Stephan Mohrdieck · Robert Wendt

\title{
Integral conjugacy classes of compact Lie groups
}

Received: 2 July 2003 / Revised Version: 14 January 2004

Published online: 3 March 2004

\begin{abstract}
We show that untwisted respectively twisted conjugacy classes of a compact and simply connected Lie group which satisfy a certain integrality condition correspond naturally to irreducible highest weight representations of the corresponding affine Lie algebra. Along the way, we review the classification of twisted conjugacy classes of a simply connected compact Lie group $G$ and give a description of their stabilizers in terms of the Dynkin diagram of the corresponding twisted affine Lie algebra.
\end{abstract}

\section{Introduction}

Let $G$ be a group and $\tau \in A u t(G)$ an automorphism of $G$. The $\tau$-twisted conjugacy classes of $G$ are the orbits of the action of $G$ on itself which is given by $g: h \mapsto g h \tau\left(g^{-1}\right)$. We shall always allow $\tau$ to be the identity in which case the $\tau$-twisted conjugacy classes of $G$ are just the ordinary conjugacy classes of $G$.

In this note, we study twisted and untwisted conjugacy classes of a compact simply connected simple Lie group $G$ which satisfy a certain integrality condition. This condition appears in the physics literature where it is used in the classification of so called D-branes in the Wess-Zumino-Witten model $[4,11]$. In a mathematical context, integral conjugacy classes play a role in the study of gerbes on compact Lie groups [14]. Furthermore, the integrality condition for conjugacy classes in the group $G$ (which we will state in equation (1) below) can be seen as an analogue of the integrality condition for coadjoint orbits of $G$. It is well known that, via the Borel-Weil-Bott construction, integral coadjoint orbits of $G$ correspond to irreducible representations of $G$. On the other hand, it has been observed in [14] that untwisted integral conjugacy classes in $G$ are parametrized by the same set as irreducible highest weight representations of the affine Lie algebra corresponding to $G$. This observation has been extended in [18] to the case of twisted conjugacy classes of $S U$ (3) (where the twisting automorphism comes from the diagram automorphism of the Dynkin diagram of $S U(3)$ ), and irreducible highest weight representations of the corresponding twisted affine Lie algebra.

S. Mohrdieck: Universität Basel, Rheinsprung 21, 4051 Basle, Switzerland. e-mail: mohrdis@math.unibas.ch

R. Wendt: University of Toronto, Department of Mathematics, 100 St.George Street, Toronto, Ontario M5S 3G3, Canada. e-mail: rwendt@math.toronto.edu

Mathematics Subject Classification (2000): 22E46, 22E67, 81R10 
The main goal of this note is to extend the correspondence between integral conjugacy classes and irreducible highest weight representations to twisted conjugacy classes of arbitrary simple compact and simply connected Lie groups and to give a geometric reason for this correspondence which works for both twisted and untwisted conjugacy classes simultaneously. This is achieved by translating the integrality condition for untwisted respectively twisted conjugacy classes in a compact Lie group $G$ to an integrality condition for coadjoint orbits of the corresponding untwisted respectively twisted loop group of $G$. The correspondence to irreducible highest weight representations then comes naturally from the ideas of geometric quantization (or, equivalently, Kirillov's orbit method) which relates certain coadjoint orbits of a Lie group to its unitary representations. Along the way, we review some standard facts about conjugacy classes in compact Lie groups and extend them to the case of twisted conjugacy classes. A rather algebraic approach relating twisted conjugacy classes to irreducible highest weight representations of twisted affine Lie algebras via boundary conformal field theory has been developed in $[5,10]$.

The contents of this note is as follows: In section 2, we review some basic facts about twisted conjugation. In section 3, we explicitly describe the set of twisted conjugacy classes of a compact simply connected Lie group $G$ as a convex polytope in an Euclidean vector space whose faces of maximal dimension are in one to one correspondence with the vertices of a certain twisted affine Dynkin diagram. In fact, this polytope can naturally be identified with the fundamental domain of the twisted affine Weyl group corresponding to $G$ and an automorphism $\tau$ of $G$. This generalizes the classical situation of ordinary conjugacy classes which are parametrized by the fundamental alcove of the affine Weyl group corresponding to $G$. In section 4, we show how to calculate the stabilizers of the twisted conjugacy classes. Since we chose $G$ to be simply connected, the stabilizers of twisted conjugacy classes are connected subgroups of $G$. The Dynkin diagram of the Lie algebras of the stabilizers turn out to be exactly the sub-diagrams of the twisted affine Dynkin diagram described in section 3. Again, this is well known in the untwisted case. The fundamental groups of the stabilizers can be easily calculated from the root data so that we have a complete description of the stabilizers.

Finally, section 5 contains the main results of this paper. Suppose, the group $G$ is simple and simply connected. Let $\eta$ denote the Cartan 3-form on $G$. That is, $\eta$ is a left invariant 3-form on $G$ which generates $H^{3}(G, \mathbb{Z})$. Let $\mathcal{C}$ denote an untwisted or twisted conjugacy class in $G$, and let $\iota: \mathcal{C} \rightarrow G$ denote the inclusion. It is known that the 3 -form $\iota^{*} \eta$ on $\mathcal{C}$ is exact. Hence we can choose a 2 -form $\varpi$ on $\mathcal{C}$ such that $d \varpi=\iota^{*} \eta$. Finally, fix some $a \in \mathbb{R}^{*}$. We call an untwisted or twisted conjugacy classes $\mathcal{C} \subset G$ integral at level $a$, if the integral

$$
a \int_{N} \eta-a \int_{\partial N} \varpi
$$

takes values in $\mathbb{Z}$ for all 3-cycles $N \subset G$ with $\partial N \subset \mathcal{C}$. In other words, we call a conjugacy class $\mathcal{C}$ integral at level $a$, if the relative 3-cocycle $a(\eta, \varpi)$ defines an integral element of the relative cohomology group $H^{3}(G, \mathcal{C})$ 
It is not hard to see that integral conjugacy classes at level $a$ can only exist if $a$ itself is an integer. Furthermore, there is a well known correspondence between (twisted) conjugacy classes in $G$ and coadjoint orbits of the corresponding (twisted) loop group $L(G)[9,19]$. Let $\Omega G$ denote the space of based loops in $G$. Using a modified version of the transgression homomorphism $\sigma: H^{3}(G) \rightarrow H^{2}(\Omega G)$ we can pull back the relative 3-cocycle $a(\eta, \varpi)$ to a 2-cocycle on the coadjoint orbit of $L(G)$ which corresponds to the twisted conjugacy class $\mathcal{C}$. It turns out that this 2-cocycle is in the same cohomology class as the standard symplectic structure on the coadjoint orbit. This allows to translate the integrality condition (1) for a conjugacy class $\mathcal{C}$ to an integrality condition for the natural symplectic form on the corresponding coadjoint orbit of $L(G)$. It is known $[9,16,19]$, that integral coadjoint orbits of a (twisted) loop group correspond naturally to integrable irreducible highest weight representations of the corresponding affine Lie algebra. So we get a natural one-to-one correspondence between integral conjugacy classes of the group $G$ at non-negative level and integrable irreducible highest weight representations of the corresponding affine Lie algebra.

\section{Twisted conjugation}

Let $G$ be a Lie group and let $\tau$ be an automorphism of $G$. The $\tau$-twisted conjugacy classes of $G$ are the orbits of the following action of the group $G$ on itself:

$$
\begin{gathered}
G \times G \rightarrow G, \\
(g, h) \mapsto g h \tau\left(g^{-1}\right) .
\end{gathered}
$$

Let $\tau^{\prime}$ be another automorphism of $G$ which differs from $\tau$ by an inner automorphism. That is, $\tau^{\prime}(g)=\tau\left(u g u^{-1}\right)$ for some $u \in G$. Then the map $h \mapsto u h$ maps $\tau^{\prime}$-twisted conjugacy classes in $G$ to $\tau$-twisted conjugacy classes and induces an diffeomorphism between the corresponding twisted conjugacy classes. From this point of view, it is enough to consider automorphisms $\tau$ up to inner automorphisms of $G$.

Now let us suppose that $G$ is compact and semi-simple. Let $\operatorname{Aut}(G)$ denote the group of automorphisms of $G$, and let $\operatorname{Int}(G)$ denote the subgroup of inner automorphisms. Then $\operatorname{Aut}(G) / \operatorname{Int}(G)$ is a finite group. After fixing a maximal torus $T \subset G$ and a basis $\Pi$ of the root system $\Delta$ of $G$ with respect to $T$, the group $\operatorname{Aut}(G) / \operatorname{Int}(G)$ can be identified with a subgroup of the group of graph-automorphisms of the Dynkin diagram of $\Delta$. In fact, if we assume $G$ to be simply connected, then the group $\operatorname{Aut}(G) / \operatorname{Int}(G)$ is isomorphic to the group of graph-automorphisms of the Dynkin diagram. This observation allows us to find a nice representative in each connected component of $\operatorname{Aut}(G)$. Namely, after fixing a maximal torus $T \subset G$ and a basis $\Pi$ of the root system $\Delta$ of $G$, any graph-automorphisms of the Dynkin diagram corresponds to a permutation of the set $\Pi$ and can be lifted to an automorphism of $G$ leaving $T$ invariant. From now on, we will only consider automorphisms $\tau$ of $G$ which come from this construction.

One can view twisted conjugacy classes as $G$-orbits in the non-connected Lie group $\widetilde{G}=G \rtimes \operatorname{Aut}(G) / \operatorname{Int}(G)$, where $G$ acts on $\widetilde{G}$ by conjugation. From this 
point of view, the twisted conjugacy classes have been studied in [17] (see also [15] and [19]).

\section{The space of conjugacy classes}

Our first goal is to describe the set of $\tau$-twisted conjugacy classes in $G$. Choosing $\tau$ to be the identity yields a description of the set of ordinary conjugacy classes in $G$. Since $\tau$ leaves the maximal torus $T \subset G$ invariant, we can consider the subgroup $T^{\tau} \subset T$ of $\tau$-invariants. This group will in general not be connected. Let us denote by $T_{0}^{\tau}$ the connected component of $T^{\tau}$ containing the identity. It is a fact that every $\tau$-twisted conjugacy class in $G$ intersects $T_{0}^{\tau}$ in at least one point (see e.g. [7], Proposition 4.3).

So in order to describe the set of $\tau$-twisted conjugacy classes in $G$, it remains to check, which elements of $T_{0}^{\tau}$ are twisted conjugate under $G$. To this end, let us introduce the twisted Weyl group

$$
W(G, T, \tau)=N_{G}^{\tau}\left(T_{0}^{\tau}\right) / T_{0}^{\tau} .
$$

Here, $N_{G}^{\tau}\left(T_{0}^{\tau}\right)=\left\{g \in G \mid g T_{0}^{\tau} \tau(g)^{-1}=T_{0}^{\tau}\right\}$ denotes the normalizer of $T_{0}^{\tau}$ with respect to $\tau$-twisted conjugation. It is a general fact that $W(G, T, \tau)$ is a finite group (see e.g.[7]). The twisted Weyl group $W(G, T, \tau)$ can be seen as a generalization of the ordinary Weyl group $N(G, T)=N_{G}(T) / T$ of $G$ with respect to the maximal torus $T$. One can show that two elements of $T_{0}^{\tau}$ are twisted conjugate under $G$ if and only if they are conjugate under $W(G, T, \tau)$. So the space of $\tau$-twisted conjugacy classes in $G$ can be identified with the quotient $T_{0}^{\tau} / W(G, T, \tau)$. We shall now describe this quotient in more detail.

Let $W=N_{G}(T) / T$ denote the usual Weyl group of $G$. The action of $\tau$ on the torus $T$ induces an action of $\tau$ on the Weyl group $W(G, T)$. Let $W^{\tau}$ denote the subgroup of $W(G, T)$ which consists of elements commuting with $\tau$. We also introduce the finite group $\left(T / T_{0}^{\tau}\right)^{\tau}$. Then one obtains the following isomorphism $[15,19]$ :

$$
W(G, T, \tau) \cong W(G, T)^{\tau} \ltimes\left(T / T_{0}^{\tau}\right)^{\tau} .
$$

The group $W^{\tau}$ is the Weyl group of the identity component of the fixed point group $G^{\tau}$. Since $T_{0}^{\tau}$ is a maximal torus of this group and every element of $W^{\tau}$ commutes with $\tau$, the group $W^{\tau}$ acts on $T_{0}^{\tau}$ by its Weyl group action on $T_{0}^{\tau}$. Now let us study the action of $\left(T / T_{0}^{\tau}\right)^{\tau}$ on $T_{0}^{\tau}$. Take some element $\bar{t} \in\left(T / T_{0}^{\tau}\right)^{\tau}$ and fix a pre-image $t$ of $\bar{t}$ under the projection $T \rightarrow T / T_{0}^{\tau}$. The condition that $\bar{t}$ is invariant under $\tau$ translates to the equation $\tau(t)=t s$ for some $s \in T_{0}^{\tau}$. Hence $t t_{0} \tau\left(t^{-1}\right)=t_{0} s$ for all $t_{0} \in T^{\tau}$. Thus, $T / T_{0}^{\tau}$ acts on $T_{0}^{\tau}$ by translations.

The group $T / T_{0}^{\tau}$ and its action on $T_{0}^{\tau}$ can be described more explicitly: Let $\mathfrak{h}$ denote the Lie algebra of $T$. The Killing form on $G$ induces a $W(G, T)$-invariant inner product on $\mathfrak{h}$ which is also invariant under the action of $\tau$ on $\mathfrak{h}$. Let $\mathfrak{h}^{\tau}$ denote the $\tau$-invariant part of $\mathfrak{h}$, i.e. the Lie algebra of $T_{0}^{\tau}$ and let $\pi: \mathfrak{h} \rightarrow \mathfrak{h}^{\tau}$ denote the orthogonal projection with respect to the Killing form on $\mathfrak{h}$. Finally, let $\exp : \mathfrak{h} \rightarrow T$ denote the exponential map. Its kernel is a lattice in $\mathfrak{h}$ and we can identify $T$ with $\mathfrak{h} / \operatorname{ker}(\exp )$. Since $\tau$ acts on $T$, its induced action on $\mathfrak{h}$ leaves the 
lattice $\operatorname{ker}(\exp )$ invariant so that we have $T_{0}^{\tau}=\mathfrak{h}^{\tau} / \operatorname{ker}(\exp )^{\tau}$. Finally, one checks directly that $\left(T / T_{0}^{\tau}\right)^{\tau} \cong \pi(k \operatorname{er}(\exp )) / \operatorname{ker}(\exp )^{\tau}$ and that the translation action of $\left(T / T_{0}^{\tau}\right)^{\tau}$ on $T_{0}^{\tau}$ comes from the translation action of $\pi(k e r(\exp ))$ on $\mathfrak{h}^{\tau}$.

The observations above allow us to identify the space of twisted conjugacy classes with the set $\mathfrak{h}^{\tau} / W^{\tau} \ltimes \pi(k \operatorname{er}(\exp ))$. This set can be identified with a polytope in $\mathfrak{h}^{\tau}$ as follows. First, let $K \subset \mathfrak{h}$ denote the fundamental Weyl chamber $K=$ $\{h \in \mathfrak{h} \mid \alpha(h)>0$ for all $\alpha \in \Pi\}$. Then $K \cap \mathfrak{h}^{\tau} \neq \emptyset$ and we have $K \cap \mathfrak{h}^{\tau}=\{h \in$ $\mathfrak{h}^{\tau}|\alpha|_{\mathfrak{h}^{\tau}}(h)>0$ for all $\left.\alpha \in \Pi\right\}$. The closure $\bar{K}=\{h \in \mathfrak{h} \mid \alpha(h) \geq 0$ for all $\alpha \in \Pi\}$ of $K$ is a fundamental domain for the action of the Weyl group $W$ of $G$ on $\mathfrak{h}$. Similarly, the closure $\bar{K} \cap \mathfrak{h}^{\tau}$ is a fundamental domain for the action of $W^{\tau}$ on $\mathfrak{h}^{\tau}$.

It remains to find a fundamental domain for the action of the group $W^{\tau} \ltimes$ $\pi(k e r(\exp ))$ on $\mathfrak{h}^{\tau}$. It is well known that if the group $G$ is simply connected and for $\tau=i d$, the lattice $\operatorname{ker}(\exp )$ is the dual root lattice of $G$. Hence, the group $W \ltimes \operatorname{ker}(\exp )$ is the affine Weyl group of $\mathfrak{g}$, or equivalently the Weyl group of the affine Kac-Moody algebra corresponding to $\mathfrak{g}$. A fundamental domain for the action of $W \ltimes k e r(\exp )$ is given by the fundamental alcove, i.e. by the set

$$
\mathfrak{a}=\{h \in \mathfrak{h} \mid \alpha(h) \geq 0 \text { for all } \alpha \in \Pi \text { and } \theta(h) \leq 1\} .
$$

Here, $\theta \in \Delta$ denotes the highest root of $\Delta$ with respect to $\Pi$. If $G$ is not simply connected, we have to divide $\mathfrak{h}$ by $W \ltimes \Lambda$, where $\Lambda \subset \mathfrak{h}$ denotes the lattice of all smooth homomorphisms $S^{1} \rightarrow T$.

We now want a similar description for a fundamental domain for the action of $W^{\tau} \ltimes \pi(k e r(\exp ))$ on $\mathfrak{h}^{\tau}$. First, we have to make a general observation. Denote by $\Delta^{\tau}$ the set $\Delta^{\tau}=\left\{\left.\alpha\right|_{\mathfrak{h}}{ }^{\tau} \mid \alpha \in \Delta\right\}$. This is a subset in $\left(\mathfrak{h}^{\tau}\right)^{*}$, and if $\Delta$ is not of type $\mathrm{A}_{2 n}$, the set $\Delta^{\tau}$ is a root system. If $\Delta$ is of type $A_{2 n}$ with $n>1$, then $\Delta^{\tau}$ is a non-reduced root system of type $B C_{n}$, i.e. it is built out of the root system $B_{n}$ and $C_{n}$ such that the long roots of $B_{n}$ are the short roots of $C_{n}$. In the case $\Delta=A_{2}$, the set $\Delta^{\tau}$ consists of the union of two root systems of type $A_{1}$ such that each element in one copy of $A_{1}$ is two times an element of the other copy of $A_{1}$.

For simply connected $G$ we use the observation [19] that the group $W^{\tau} \ltimes$ $\pi(k e r(\exp ))$ is the Weyl group of the twisted affine Lie algebra corresponding to $\mathfrak{g}$ and the automorphism $\tau$. A fundamental domain for the action of this group on $\mathfrak{h}^{\tau}$ can be described as follows: If $\Delta$ is not of type $A_{2 n}$, let $\theta_{\tau}$ denote the highest short root of $\Delta^{\tau}$ with respect to the basis $\left\{\left.\alpha\right|_{\mathfrak{h}^{\tau}} \mid \alpha \in \Pi\right\}$. If $\Delta$ is of type $A_{2 n}$ with $n>1$, let $\theta_{\tau}$ denote the highest short root of the subsystem $B_{n}$ of $B C_{n}$ multiplied by two (i.e. $\theta_{\tau}$ is a long root of the system $C_{n}$ ). If $\Delta$ is of type $A_{2}$, let $\theta_{\tau}$ denote the unique positive long root of $\Delta^{\tau}$.

Now, in all cases, a fundamental domain for the action of the group $W^{\tau} \ltimes$ $\pi(\operatorname{ker}(\exp ))$ on $\mathfrak{h}^{\tau}$ is given by the set

$$
\mathfrak{a}_{\tau}=\left\{h \in \mathfrak{h}^{\tau}|\alpha|_{\mathfrak{h}^{\tau}}(h) \geq 0 \text { for } \alpha \in \Pi \text { and } \theta_{\tau}(h) \leq \frac{1}{\operatorname{ord}(\tau)}\right\} .
$$

A proof of this fact can be found e.g. in [13], chapter 6. (Note that we have used a different normalization of the invariant bilinear form on the twisted affine Lie algebra than the one used in [13].) Again, if $G$ is not simply connected, we have to divide $\mathfrak{h}^{\tau}$ by the action of $W^{\tau} \ltimes \pi(\Lambda)$. 
Example 3.1. Let $G=S U(n)$ be the special unitary group. A maximal torus $T \subset$ $S U(n)$ is given by the set of diagonal matrices. The exponential map

$$
\exp :\left(x_{1}, \ldots x_{n}\right) \mapsto \operatorname{diag}\left(e^{2 \pi i x_{1}}, \ldots, e^{2 \pi i x_{n}}\right),
$$

identifies the Lie algebra of $T$ with the set $\left\{\left(x_{1}, \ldots x_{n}\right) \in \mathbb{R}^{n} \mid \sum x_{j}=0\right\}$. One can check directly, that the set of conjugacy classes in $S U(n)$ is parametrized by the fundamental alcove

$$
\mathfrak{a}=\left\{\left(x_{1}, \ldots, x_{n}\right) \in \mathbb{R}^{n} \mid x_{1} \geq \ldots \geq x_{n}, \quad \sum_{i} x_{i}=0, \text { and } x_{1}-x_{n} \leq 1\right\} .
$$

Example 3.2. For $n \geq 3$, The group $S U(n)$ admits a non-trivial outer automorphism $\tau$ which is defined as follows. Let $J_{n}$ denote the matrix $J_{n}=\operatorname{antidiag}(1, \ldots, 1)$ if $n$ is odd and $J_{n}=\operatorname{antidiag}(1, \ldots, 1,-1, \ldots,-1)$ if $n$ is even. Then we can set $\tau(A)=J_{n} \bar{A} J_{n}^{-1}$ for $A \in S U(n)$. The automorphism $\tau$ acts on $\mathfrak{h}$ via $\tau$ : $\operatorname{diag}\left(x_{1}, \ldots, x_{n}\right) \mapsto \operatorname{diag}\left(-x_{n}, \ldots,-x_{1}\right)$. Hence $\mathfrak{h}^{\tau}=\left\{\operatorname{diag}\left(x_{1}, \ldots, x_{n}\right) \mid x_{i}=\right.$ $-x_{n+1-i}$ for $\left.1 \leq i \leq n\right\}$. If $n=2 m$ is even, the polytope $\mathfrak{a}_{\tau}$ which parametrizes the set of $\tau$-twisted conjugacy classes in $S U(n)$ is given by

$$
\mathfrak{a}_{\tau}=\left\{\left(x_{1}, \ldots, x_{m}\right) \in \mathbb{R}^{m} \mid x_{1} \geq \ldots \geq x_{m} \geq 0, \text { and } x_{1}+x_{m} \leq \frac{1}{2}\right\} .
$$

If $n=2 m+1$ is odd and $m>1$, the polytope $\mathfrak{a}_{\tau}$ is given by

$$
\mathfrak{a}_{\tau}=\left\{\left(x_{1}, \ldots, x_{m}\right) \in \mathbb{R}^{m} \mid x_{1} \geq \ldots \geq x_{m} \geq 0, \text { and } x_{1} \leq \frac{1}{4}\right\} .
$$

Finally, in the case $S U(3)$, we have $\mathfrak{h}^{\tau}=\{(x, 0,-x) \mid x \in \mathbb{R}\}$, and

$$
\mathfrak{a}_{\tau}=\left\{x \in \mathbb{R} \mid 0 \leq x \leq \frac{1}{4}\right\} .
$$

\section{Stabilizers}

Throughout this section, let $G$ be simply connected. Given an element $h \in G$, the conjugacy class containing $h$ is isomorphic to $G / \operatorname{Stab}_{G}(h)$, where $\operatorname{Stab}_{G}(h)=$ $\left\{g \in G \mid g h \tau\left(g^{-1}\right)=h\right\}$ denotes the stabilizer of $h$ in $G$. The aim of this section is to give an explicit description of the stabilizers. We first describe the Lie algebras of the stabilizers. Using this description along with some general facts of the theory of compact Lie groups, we can calculate the fundamental groups of the stabilizers. The fact that $G$ is a simply connected compact Lie group implies that all stabilizers are connected. So we have a complete description of the twisted conjugacy classes in $G$.

As in section 2, let $\tau$ denote an automorphism of $G$ which leaves a maximal torus $T \subset G$ invariant and induces an automorphism of the corresponding Dynkin diagram of $G$. Let $\mathfrak{a} \subset \mathfrak{h}$ denote the polytope parametrizing the set of conjugacy classes in $G$, and let $\mathfrak{a}_{\tau}$ denote the polytope in $\mathfrak{h}^{\tau}$ parametrizing the set of $\tau$-twisted conjugacy classes in $G$. 
Let us set $\widetilde{\Pi}=\Pi \cup\{-\theta\}$, where, as before, $\Pi$ denotes a basis of $\Delta$. Then $\widetilde{\Pi}$ are the vertices of the extended Dynkin diagram of $\Delta$ or equivalently, the Dynkin diagram of the affine Lie algebra corresponding to $G$. Similarly, the set $\widetilde{\Pi}^{\tau}=$ $\Pi^{\tau} \cup\left\{-\theta_{\tau}\right\}$ labels the vertices of the Dynkin diagram of the twisted affine Lie algebra corresponding to $G$ and the automorphism $\tau$ of $G$.

It is implicitly contained in the classical literature on compact Lie groups (see e.g. [1]), that the stabilizers of elements of $G$ under ordinary conjugation have Dynkin diagrams which can be obtained by deleting vertices from the Dynkin diagram corresponding to $\widetilde{\Pi}$. This fact is a special case of the following proposition in the case $\tau=i d{ }^{1}$

Proposition 4.1. Let $G$ act on itself by $\tau$-twisted conjugation and let $H \in \mathfrak{a}_{\tau}$. Then the Dynkin diagram of the Lie algebra of $\operatorname{Stab}(\exp (H))$ is the sub-diagram of the Dynkin diagram corresponding to $\widetilde{\Pi}^{\tau}$ which is obtained by deleting all $\alpha$ from the finite Dynkin diagram $\Pi^{\tau}$ for which $\alpha(H) \notin \mathbb{Z}$ and deleting the vertex corresponding to $\theta_{\tau}$ whenever $\theta_{\tau}(H) \notin \frac{1}{\operatorname{ord}(\tau)}+\mathbb{Z}$.

The Lie algebra of Stab $(\exp (H))$ is given by the sum of $\mathfrak{h}^{\tau}$ and the sub-algebra of $\mathfrak{g}$ corresponding to the diagram described above.

Proof. Let us start with the case that $\mathfrak{g}$ is not of type $A_{2 m}$. The group $\operatorname{Stab}_{G}(\exp (H))$ can be written as

$$
\operatorname{Stab}_{G}(\exp (H))=\{g \in G \mid \exp (H) \tau(g) \exp (-H)=g\} .
$$

Therefore its Lie algebra is given by

$$
\operatorname{Lie}\left(\operatorname{Stab}_{G}(\exp (H))\right)=\{X \in \mathfrak{g} \mid \operatorname{Ad}(\exp (H)) \circ \tau(X)=X\} .
$$

We can decompose the Lie algebra $\mathfrak{g}_{\mathbb{C}}$ into the eigenspaces of $\tau$ :

$$
\mathfrak{g}_{\mathbb{C}}=\bigoplus_{k=0}^{\operatorname{ord}(\tau)-1} \mathfrak{g}_{k}
$$

where $\mathfrak{g}_{k}$ denotes the $e^{2 \pi i \frac{k}{\operatorname{ord}(\tau)}}$-eigenspace of the action of $\tau$ on $\mathfrak{g}_{\mathbb{C}}$. It is known that $\mathfrak{g}_{0}$ is a semi-simple Lie algebra with root system $\Delta^{\tau}$ and that $\mathfrak{g}_{k}$ with $k \neq 0$ are representations of $\mathfrak{g}_{0}$ whose highest weight is given by $\theta_{\tau}$, the highest short root of $\Delta^{\tau}$. The Cartan sub-algebra of $\mathfrak{g}_{0}$ is given by $\mathfrak{h}_{\mathbb{C}}^{\tau}$. Decomposing $\mathfrak{g}_{k}$ into weight spaces with respect to $\mathfrak{h}^{\tau}$, we can write

$$
\mathfrak{g}_{\mathbb{C}}=\mathfrak{h}^{\tau} \oplus \bigoplus_{\alpha \in \Delta^{\tau}} \mathfrak{g}_{\alpha} \oplus \bigoplus_{k=1}^{\operatorname{ord}(\tau)-1} \bigoplus_{\lambda \in P_{k}} \mathfrak{g}_{\lambda},
$$

where $P_{k} \subset\left(\mathfrak{h}^{\tau}\right)^{*}$ denotes the set of weights of $\mathfrak{g}_{k}$ as a representation of $\mathfrak{g}_{0}$

1 The method described in [12] does not give the complete set of the possible stabilizers. E.g. the group $\mathrm{B}_{4}$ appears as a stabilizer in $\mathrm{F}_{4}$, but cannot be obtained by deleting vertices of the unextended Dynkin diagram of $\mathrm{F}_{4}$. 
Let us view $X$ as an element of $\mathfrak{g} \mathbb{C}$ and write

$$
X=H_{0}+\sum_{\alpha \in \Delta^{\tau}} X_{\alpha}+\sum_{k=1}^{\operatorname{ord}(\tau)-1} \sum_{\lambda \in P_{k}} X_{\lambda},
$$

we see that we get

$$
\begin{aligned}
A d(\exp (H)) \circ \tau(X)= & H_{0}+\sum_{\alpha \in \Delta^{\tau}} e^{2 \pi i \alpha(H)} X_{\alpha} \\
& +\sum_{k=1}^{\operatorname{ord}(\tau)-1} \sum_{\lambda \in P_{k}} e^{2 \pi i \frac{k}{\operatorname{ord}(\tau)}} e^{2 \pi i \lambda(H)} X_{\lambda} .
\end{aligned}
$$

This implies that the Lie algebra of $\operatorname{Stab}(\exp (H))$ is given as the sum of $\mathfrak{h}_{\mathbb{C}}^{\tau}$ with those $\mathfrak{g}_{\alpha}$ such that $\alpha(H) \in \mathbb{Z}$ and those $\mathfrak{g}_{\lambda}$ with $\lambda \in P_{k}$ such that $\lambda(H) \in \frac{-k}{\operatorname{ord}(\tau)}+\mathbb{Z}$. Since we have chosen $H \in \mathfrak{a}_{\tau}$, and since $\theta_{\tau}$ is the highest weight of the $\mathfrak{g}_{k}$ we have $0 \leq \alpha(H) \leq 1$ and $0 \leq \lambda(H) \leq \frac{1}{\operatorname{ord}(\tau)}$ for all $\alpha \in \Delta_{\tau}$ and $\lambda \in P_{k}$. Furthermore, $|\lambda(H)|=\frac{1}{\operatorname{ord}(\tau)}$ can only be obtained for $\lambda= \pm \theta_{\tau}$ in which case $H$ has to lie on the boundary of $\mathfrak{a}_{\tau}$. If $H$ lies in the interior of $\mathfrak{a}_{\tau}$, we have $\alpha(H) \notin \mathbb{Z}$ for all $\alpha \in \Delta^{\tau}$ and $\lambda(H) \notin \frac{-k}{\operatorname{ord}(\tau)}+\mathbb{Z}$ for all $\lambda \in P_{k}$. If $H$ lies on the boundary of $\mathfrak{a}_{\tau}$, the stabilizer of $H$ is generated by the elements of $\widetilde{\Pi}^{\tau}=\Pi^{\tau} \cup\left\{\theta_{\tau}\right\}$ for which either $\alpha(H) \in \mathbb{Z}$ if $\alpha \in \Pi^{\tau}$ or $\theta_{\tau}(H) \in \frac{-k}{\operatorname{ord}(\tau)}+\mathbb{Z}$.

The case that $\mathfrak{g}$ is of type $A_{2 m}$ can be obtained by similar arguments, one just has to be more careful with the root system $\Delta^{\tau}$ which is non-reduced.

Remark 4.2. There is a second approach to Proposition 4.1 which works for semisimple conjugacy classes in algebraic groups. This approach uses the classification of finite order automorphisms of simple Lie algebras and has been worked out in the proof of Theorem 3.2 in [15].

Finally, we have to study the topology of the stabilizers. Since we assumed $G$ to be simply connected, all stabilizers are connected. So in order to describe them explicitly, we only have to determine their fundamental groups. This can be done using some standard facts from the theory of compact Lie groups (see e.g. [7]): Let $\Delta_{H} \subset\left(\mathfrak{h}^{\tau}\right)^{*}$ denote the root system of the group $\operatorname{Stab}(\exp (H))$, let $Q_{H} \subset\left(\mathfrak{h}^{\tau}\right)^{*}$ denote the lattice generated by $\Delta_{H}$.

We can use the normalized Killing form on $\mathfrak{h}$ to identify $\left(\mathfrak{h}^{\tau}\right)^{*}$ with $\mathfrak{h}^{\tau}$. Let $Q_{H}^{\vee}$ be the image of $Q_{H}$ under this embedding. Then

$$
\pi_{1}(\operatorname{Stab}(\exp (H))) \cong \operatorname{ker}(\exp )^{\tau} / Q_{H}^{\vee} .
$$

Example 4.3. In figure 1 we indicate how the constructions described in this section apply to the spaces of conjugacy classes of the simply connected compact Lie groups of type $C_{2}$ compared to the space of twisted conjugacy classes of the simply connected Lie groups of type $A_{3}$. The picture shows the fundamental domains of the respective untwisted and twisted affine Weyl groups. The faces of the fundamental domain are labeled with the stabilizers of the corresponding conjugacy classes. 

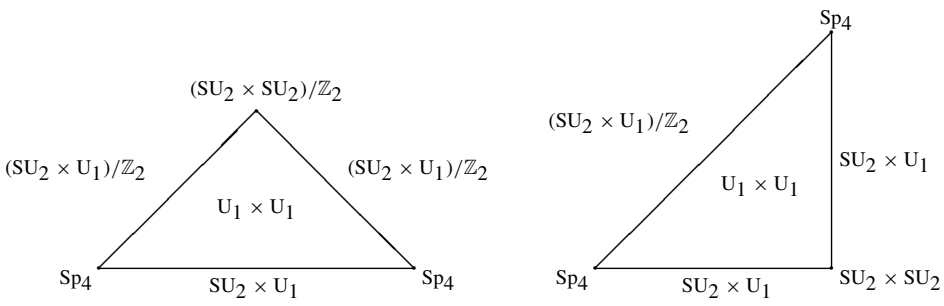

Fig. 1. Conjugacy classes of $S p(4)$ (left) versus $\tau$-twisted conjugacy classes of $S U$ (4) (right) where $\tau$ is a non-trivial outer automorphism of $S U(4)$

\section{Integral conjugacy classes}

\subsection{The integrality condition}

We start this section with our main definition: Suppose that $G$ is simply connected and simple and let $\eta$ denote the left-invariant 3-form on $G$ whose value at the identity is given by $\eta\left(X_{1}, X_{2}, X_{3}\right)=\frac{1}{8 \pi^{2}}\left\langle X_{1},\left[X_{2}, X_{3}\right]\right\rangle$. Thus, $\eta$ is a generator of $H^{3}(G, \mathbb{Z})$. Let $\tau$ be an automorphism of $G$, let $\mathcal{C}$ be a $\tau$-twisted conjugacy class on $G$ and denote by $\iota: \mathcal{C} \rightarrow G$ the embedding of $\mathcal{C}$ into $G$. There is a canonical 2-form $\varpi$ on $\mathcal{C}$, which is defined as follows. Given an element $A \in \mathfrak{g}$, denote by $A_{\mathcal{C}}$ the generating vector field of the action of $G$ on $\mathcal{C}$. Then we set

$$
\varpi_{g}\left(A_{\mathcal{C}}, B_{\mathcal{C}}\right)=\frac{1}{8 \pi^{2}}\left(\left\langle\operatorname{Ad}_{g} \circ \tau(A), B\right\rangle-\left\langle\operatorname{Ad}_{g} \circ \tau(B), A\right\rangle\right) .
$$

It is known (see e.g. [2] Proposition 2.1, [3]) that

$$
d \varpi=\iota^{*} \eta
$$

Definition 5.1. Fix some $a \in \mathbb{R}^{*}$. We call a twisted or untwisted conjugacy class $\mathcal{C} \subset G$ integral at level $a$ if the integral

$$
a \int_{N} \eta-a \int_{\partial N} \varpi
$$

takes values in $\mathbb{Z}$ for all 3-cycles $N \subset G$ with $\partial N \subset \mathcal{C}$.

In other words, we call a conjugacy class $\mathcal{C}$ integral at level $a$ if the relative cocycle $a(\eta, \varpi) \in H^{3}(G, \mathcal{C})$ is integral.

The goal of this section is to classify integral conjugacy classes in terms of integral coadjoint orbits of certain centrally extended loop groups, and thus to relate them to integrable highest weight representations of the corresponding affine Lie algebras. 


\subsection{Coadjoint orbits of loop groups}

Let $G$ be as before. We denote by $L(G)=C^{\infty}\left(S^{1}, G\right)$ the loop group of $G .^{2}$ There is a universal central extension $\widehat{L(G)}$ of the group $L(G)$ which, as a topological space, is a non-trivial $S^{1}$-bundle over $L(G)$. The underlying vector space of the Lie algebra of $\widehat{L(G)}$ is given by $\widehat{L(\mathfrak{g})}=L(\mathfrak{g}) \oplus \mathbb{R}$, where $L(\mathfrak{g})=C^{\infty}\left(S^{1}, \mathfrak{g}\right)$ denotes the loop algebra of $\mathfrak{g}$. The smooth part of the dual of $\widehat{L(\mathfrak{g})}$ can be identified with $L(\mathfrak{g}) \oplus \mathbb{R}$ via the pairing

$$
\langle(X, a),(Y, b)\rangle=\frac{1}{2 \pi} \int_{S^{1}}\langle X(\theta), Y(\theta)\rangle d \theta+a b .
$$

Here, the bilinear form $\langle$,$\rangle on the right hand denotes the Killing form on the Lie$ algebra $\mathfrak{g}$ normalized so that the long roots of $\mathfrak{g}_{\mathbb{C}}$ have square length 2 .

The coadjoint action of the loop group $L(G)$ on $L(\mathfrak{g}) \oplus \mathbb{R}$ is given by $[16,9]$

$$
\operatorname{Ad}_{g}^{*}:(X, a) \mapsto\left(g X g^{-1}-a g^{\prime} g^{-1}, a\right),
$$

where $g$ is an element of $L(G)$ and $g^{\prime}=\frac{d}{d \theta} g$ denotes the derivative of $g$ with respect to $\theta$. Let us denote by $\mathcal{O}_{(X, a)} \subset L(\mathfrak{g}) \oplus \mathbb{R}$ the $L(G)$-orbit through $(X, a)$.

For $a \neq 0$, we can solve the differential equation

$$
z^{\prime}=-\frac{1}{a} X z
$$

Let us denote by $z_{(X, a)}$ the unique solution of this equation with initial condition $z_{(X, a)}(0)=e$, where $e$ is the identity element in $G$. Then, $z_{(X, a)}$ is a path in the Lie group $G$ starting at $e$. Now, since $X \in L(\mathfrak{g})$ is periodic, we get $z_{(X, a)}(\theta+2 \pi)=$ $z_{(X, a)}(\theta) z_{(X, a)}(2 \pi)$. Taking another element $(Y, a)$ in the coadjoint orbit $\mathcal{O}_{(X, a)}$, we have $Y=g X g^{-1}-a g^{\prime} g^{-1}$ for some $g \in L(G)$. An easy calculation [16,9] shows that

$$
z_{(Y, a)}(\theta)=g(\theta) z_{(X, a)}(\theta) g(0)^{-1} .
$$

Since $g$ is periodic, we see that $z_{(X, a)}(2 \pi)$ and $z_{(Y, a)}(2 \pi)$ lie in the same conjugacy class in $G$. Furthermore, the stabilizer of $(X, a)$ in $L(G)$ is isomorphic to the stabilizer of $z_{(X, a)}(2 \pi)$ in $G$ so that we get

$$
\mathcal{O}_{(X, a)} \cong L(G) / \operatorname{Stab}_{G}\left(z_{(X, a)}(2 \pi)\right) .
$$

The stabilizers have been explicitly described in section 4 .

Now, let $\tau$ be an automorphism of $G$ of finite order $\operatorname{ord}(\tau)=r$. The twisted loop group $L(G, \tau)$ is defined as follows:

$$
L(G, \tau)=\left\{g \in C^{\infty}(\mathbb{R}, G) \mid g(\theta)=\tau(g(\theta+2 \pi))\right\} .
$$

${ }^{2}$ For technical reasons it is often more convenient to consider the Banach Lie group of maps $S^{1} \rightarrow G$ of some fixed Sobolev class $s>1 / 2$. We will ignore such subtleties throughout this note and stick with the more intuitive group of smooth loops 
As in the untwisted case, there is a universal central extension $\widehat{L(G, \tau)}$ of $L(G, \tau)$ by the circle group $S^{1}$ which, as a topological space, is a non-trivial $S^{1}$-bundle over $L(G, \tau)$. The Lie algebra of $\widehat{L(G, \tau)}$ is $\widehat{L(\mathfrak{g}, \tau)}=L(\mathfrak{g}, \tau) \oplus \mathbb{R}$, where $L(\mathfrak{g}, \tau)=\left\{X \in C^{\infty}(\mathbb{R}, \mathfrak{g}) \mid X(\theta)=\tau(X(\theta+2 \pi))\right\}$ denotes the twisted loop algebra of $\mathfrak{g}$. Again, the smooth part of the dual of $\widehat{L(\mathfrak{g}, \tau)}$ can be identified with $L(\mathfrak{g}, \tau) \oplus \mathbb{R}$ via a non-degenerate pairing

$$
\langle(X, a),(Y, b)\rangle=\frac{1}{2 \pi} \int_{0}^{2 \pi}\langle X(\theta), Y(\theta)\rangle d \theta+a b .
$$

The coadjoint action of $L(G, \tau)$ on $L(\mathfrak{g}, \tau) \oplus \mathbb{R}$ is again given by $g:(X, a) \mapsto$ $\left(g X g^{-1}-a g^{\prime} g^{-1}, a\right)$. It has been observed in [19] that the correspondence between coadjoint orbits of $L(G)$ and conjugacy classes in $G$ extends to a correspondence between coadjoint orbits of $L(G, \tau)$ and $\tau$-twisted conjugacy classes in $G$ as follows: Fix $(X, a) \in L(\mathfrak{g}, \tau) \oplus \mathbb{R}$ with $a \neq 0$ and, as before, solve the differential equation $z^{\prime}=-\frac{1}{a} X z$ with initial condition $z_{(X, a)}(0)=e$. If $(Y, a)$ lies in the same coadjoint orbit as $(X, a)$, a similar calculation as in the untwisted case shows that $z_{(X, a)}(2 \pi)$ and $z_{(Y, a)}(2 \pi)$ lie in the same $\tau$-twisted conjugacy class. Furthermore, the stabilizer of $(X, a)$ in $L(G, \tau)$ is isomorphic to the stabilizer of $z_{(X, a)}(2 \pi)$ in $G$ (where we mean the stabilizer with respect to $\tau$-twisted conjugation in $G$ ). So we have $\mathcal{O}_{(X, a)} \cong L(G, \tau) / \operatorname{Stab}\left(z_{(X, a)}(2 \pi)\right)$.

On every coadjoint orbit of a Lie group there exists a natural symplectic structure. In our situation, the corresponding 2-form $\omega$ on $\mathcal{O}_{(X, a)}$ with $a \neq 0$ is given as follows: Fix some $(Y, a) \in \mathcal{O}_{(X, a)}$ and let $A_{1}, A_{2}$ be two tangent vectors at $(Y, a)$. We can view $A_{i}$ as elements of $L(\mathfrak{g})$. Then the 2 -form $\omega$ at $(Y, a)$ evaluated at $A_{1}$ and $A_{2}$ is given by

$$
\omega_{(Y, a)}\left(A_{1}, A_{2}\right)=\frac{1}{2 \pi} \int_{S^{1}}\left\langle Y(\theta),\left[A_{1}(\theta), A_{2}(\theta)\right]\right\rangle d \theta+\frac{a}{2 \pi} \int_{S^{1}}\left\langle A_{1}^{\prime}(\theta), A_{2}(\theta)\right\rangle d \theta .
$$

For twisted loop groups the 2-form on the coadjoint orbits is given by the same formula.

\subsection{Integral conjugacy classes}

As we have seen in the last section, we can identify each element $(Y, a) \in \mathcal{O}_{(X, a)}$ with the unique solution of the differential equation $z^{\prime}=-\frac{1}{a} Y z$ with initial condition $z(0)=e$. This allows to define a map

$$
F: \mathbb{R} \times \mathcal{O}_{(X, a)} \rightarrow G
$$

via

$$
F:(\theta,(Y, a)) \mapsto z_{(Y, a)}(\theta) .
$$

As before, let $\eta$ denote the closed left-invariant 3-form on $G$ whose value at the identity element of $G$ evaluated on three tangent vectors $X_{1}, X_{2}, X_{3} \in \mathfrak{g}$ is given by $\eta\left(X_{1}, X_{2}, X_{3}\right)=\frac{1}{8 \pi^{2}}\left\langle X_{1},\left[X_{2}, X_{3}\right]\right\rangle$. 
Using the map $F$, we can pull back $\eta$ to a 3 -form $F^{*} \eta$ on $\mathbb{R} \times \mathcal{O}_{(X, a)}$. Its value at $(\theta,(Y, a)) \in \mathbb{R} \times \mathcal{O}_{(X, a)}$ evaluated on a triple $\left(\delta \theta, \delta_{1}(Y, a), \delta_{2}(Y, a)\right)$ of tangent vectors at $(\theta,(Y, a))$ is

$$
\frac{1}{8 \pi^{2}}\left\langle z_{(Y, a)}^{\prime}(\theta) z_{(Y, a)}(\theta)^{-1},\left[\xi_{1}(\theta), \xi_{2}(\theta)\right]\right\rangle,
$$

where we have identified the tangent vectors $\delta_{i}(Y, a)$ with vector fields $\delta_{i} z_{(Y, a)}$ along $z_{(Y, a)}$ and have set $\xi_{i}(\theta)=\delta_{i} z_{(Y, a)}(\theta) z_{(Y, a)}(\theta)^{-1}$. In particular, it follows from equation (4) that in this identification, the value of the generating vector field $B_{\mathcal{O}}$ corresponding to an element $B \in L(\mathfrak{g})$ at $z=z_{(Y, a)}$ is given by

$$
B_{\mathcal{O}}(z)=\left(r_{z}\right)_{*} B-\left(l_{z}\right)_{*} B(0),
$$

Where $r_{g}$ and $l_{g}$ denote right and left translation by an element $g \in G$. In particular, we find

$$
\xi_{B}(\theta)=B(\theta)-A d_{z(\theta)} B(0) .
$$

Now we can integrate the form $F^{*} \eta$ over $[0,2 \pi]$ to obtain a 2-form

$$
\sigma(\eta)=\int_{0}^{2 \pi} F^{*} \eta(\theta) d \theta
$$

on the coadjoint orbit $\mathcal{O}_{(X, a)}$.

Let $\mathcal{C}$ denote the (twisted) conjugacy class corresponding to the coadjoint orbit $\mathcal{O}_{(X, a)}$. We can define a map $F_{2 \pi}: \mathcal{O}_{(X, a)} \rightarrow \mathcal{C}$ via

$$
F_{2 \pi}(Y, a)=z_{(Y, a)}(2 \pi)
$$

and use this map to pull back the 2-form $\varpi$ on $\mathcal{C}$ to a 2 -form $F_{2 \pi}^{*} \varpi$ on $\mathcal{O}_{(X, a)}$. It is easy to see that the two form $\sigma(\eta)-F_{2 \pi}^{*} \varpi$ is closed.

Proposition 5.2. Let $a \neq 0$. Then the 2-form $a \sigma(\eta)-a F_{2 \pi}^{*} \varpi$ on the coadjoint orbit $\mathcal{O}_{(X, a)}$ is co-homologous to a multiple $\frac{1}{2 \pi} \omega$ of the Kirillov-Kostant symplectic form on the coadjoint orbit $\mathcal{O}_{(X, a)}$.

Proof. We have to compare the two form $2 \pi a\left(\sigma(\eta)-F_{2 \pi}^{*} \varpi\right)$ with the symplectic form $\omega$. To this end, let us introduce a 1 -form $\beta$ on $\mathcal{O}_{(X, a)}$, which, at a point $(Y, a) \in \mathcal{O}_{(X, a)}$ evaluated at a tangent vector $\delta(Y, a)$ is given by

$$
\beta_{(Y, a)}(\delta(Y, a))=\frac{a}{4 \pi} \int_{0}^{2 \pi}\left\langle z_{(Y, a)}^{\prime}(\theta) z_{(Y, a)}(\theta)^{-1}, \xi(\theta)\right\rangle d \theta
$$

with $\xi$ as before. Since we have

$$
\xi_{i}\left(\beta\left(\delta_{j}(Y, a)\right)(Y, a)=\frac{a}{4 \pi} \int_{0}^{2 \pi}\left\langle\xi_{i}^{\prime}(\theta), \xi_{j}(\theta)\right\rangle d \theta\right.
$$

and

$$
z_{(Y, a)}^{\prime}(\theta) z_{(Y, a)}(\theta)^{-1}=-\frac{1}{a} Y(\theta)
$$


we find

$$
\begin{aligned}
d \beta_{(Y, a)}\left(\xi_{1}, \xi_{2}\right)= & \frac{1}{4 \pi} \int_{0}^{2 \pi}\left\langle Y(\theta),\left[\xi_{1}(\theta), \xi_{2}(\theta)\right]\right\rangle d \theta \\
& +\frac{a}{2 \pi} \int_{0}^{2 \pi}\left\langle\xi_{1}^{\prime}(\theta), \xi_{2}(\theta)\right\rangle d \theta-\frac{a}{4 \pi}\left\langle\xi_{1}(2 \pi), \xi_{2}(2 \pi)\right\rangle
\end{aligned}
$$

and hence

$$
\begin{aligned}
2 \pi a \sigma(\eta)\left(\xi_{1}, \xi_{2}\right)+d \beta\left(\xi_{1}, \xi_{2}\right)= & \frac{1}{2 \pi} \int_{0}^{2 \pi}\left\langle Y(\theta),\left[\xi_{1}(\theta), \xi_{2}(\theta)\right]\right\rangle d \theta \\
& +\frac{a}{2 \pi} \int_{0}^{2 \pi}\left\langle\xi_{1}^{\prime}(\theta), \xi_{2}(\theta)\right\rangle d \theta \\
& -\frac{a}{4 \pi}\left\langle\xi_{1}(2 \pi), \xi_{2}(2 \pi)\right\rangle .
\end{aligned}
$$

Since we can assume the $\xi_{i}$ to come from generating vectorfields of the $L(G)$ action, we can write $\xi_{i}(\theta)=B_{i}(\theta)-A d_{z(\theta)} B_{i}(0)$ for some $B_{i} \in L(\mathfrak{g})$ (respectively $B_{i} \in L(\mathfrak{g}, \tau)$ in the twisted case) and $z=z_{(Y, a)}$. Inserting this into the equation above, a short calculation gives

$$
\begin{aligned}
2 \pi a \sigma(\eta)\left(\xi_{1}, \xi_{2}\right)+d \beta\left(\xi_{1}, \xi_{2}\right) & \\
= & \frac{1}{2 \pi} \int_{0}^{2 \pi}\left\langle Y(\theta),\left[B_{1}(\theta), B_{2}(\theta)\right]\right\rangle d \theta+\frac{a}{2 \pi} \int_{0}^{2 \pi}\left\langle B_{1}^{\prime}(\theta), B_{2}(\theta)\right\rangle d \theta \\
& +\frac{a}{4 \pi}\left\langle A d_{z(2 \pi)} B_{1}(0), B_{2}(2 \pi)\right\rangle-\frac{a}{4 \pi}\left\langle A d_{z(2 \pi)} B_{2}(0), B_{1}(2 \pi)\right\rangle .
\end{aligned}
$$

Recall that $B_{i}(0)=\tau\left(B_{i}(2 \pi)\right)$, (with $\tau=i d$ in the untwisted case) so that we have

$$
\frac{a}{4 \pi}\left\langle A d_{z(2 \pi)} B_{1}(0), B_{2}(2 \pi)\right\rangle-\frac{a}{4 \pi}\left\langle A d_{z(2 \pi)} B_{2}(0), B_{1}(2 \pi)\right\rangle=2 \pi a F_{2 \pi}^{*} \varpi\left(\xi_{1}, \xi_{2}\right)
$$

Hence we get

$$
2 \pi a \sigma(\eta)-2 \pi a F_{2 \pi}^{*} \varpi=\omega .
$$

Our next goal is to translate the integrality condition (2) for the pair $(\mathcal{C}, a)$ to an integrality condition for the 2-form $a \sigma(\eta)-a F_{2 \pi}^{*} \varpi$, and hence for the symplectic form $\frac{1}{2 \pi} \omega$ on the corresponding coadjoint orbit $\mathcal{O}_{(X, a)}$. Given any closed 2-cycle $\widetilde{N} \in H_{2}\left(\mathcal{O}_{(X, a)}\right)$, we get a 3-cycle $N$ in $G$ by mapping $(Y, a) \in \widetilde{N}$ to $\left\{z_{(Y, a)}(\theta) \mid 0 \leq \theta \leq 2 \pi\right\}$. By the results of the last section, we have $\partial N \subset \mathcal{C}$ so that $N$ is indeed a relative 3 -cycle. Furthermore, by construction we have

$$
\int_{\widetilde{N}} \frac{1}{2 \pi} \omega=\int_{\widetilde{N}} a\left(\sigma(\eta)-F_{2 \pi}^{*} \varpi\right)=a \int_{N} \eta-a \int_{\partial N} \varpi,
$$

so that integrality of $\frac{1}{2 \pi} \omega$ is necessary for the integrality for $a \eta$. 
In the other direction, we need the following proposition.

Proposition 5.3. For any relative 3 -cycle $N$ in $G$ with $\partial N \subset \mathcal{C}$, there exists a (not necessarily unique) closed 2-cycle $\widetilde{N}^{*} \in H_{2}\left(\mathcal{O}_{(X, a)}\right)$ such that $\partial N^{*}=\partial N$. Here, the 3-cycle $N^{*}$ is obtained from $\widetilde{N}^{*}$ by the construction described above.

We will postpone the proof of proposition 5.3 to section 5.4.

Let us fix a 3-cycle $N$ in $G$ with $\partial N \subset \mathcal{C}$ and let $\widetilde{N}^{*} \in H_{2}\left(\mathcal{O}_{(X, a)}\right)$ be the 2cycle from proposition 5.3. As we have noted, the cycle $\widetilde{N}^{*}$ need not be unique, but we can do with the following. Since $\partial\left(N-N^{*}\right)=0$, we have $N-N^{*} \in H_{3}(G)$. We can write

$$
\begin{aligned}
a \int_{N} \eta-a \int_{\partial N} \varpi & =a \int_{N-N^{*}} \eta+a \int_{N^{*}} \eta-a \int_{\partial N} \varpi \\
& =a \int_{N-N^{*}} \eta+a \int_{\widetilde{N}^{*}}\left(\sigma(\eta)-F_{2 \pi}^{*} \varpi\right) .
\end{aligned}
$$

Now, let us take $N$ to be a generator of $H_{3}(G) \cong \mathbb{Z}$. Since $\partial N=\emptyset$, we find $N^{*}=0$. We have chosen $\eta$ to be the generator of $H^{3}(G, \mathbb{Z}) \cong \mathbb{Z}$ so that the integrality condition (2) translates to $a \in \mathbb{Z}$. But this implies $a \int_{N-N^{*}} \eta \in \mathbb{Z}$ for all 3-cycles $N$ in $G$ with $\partial N \subset \mathcal{C}$ so that integrality of $a \sigma(\eta)-a F_{2 \pi}^{*} \varpi$ implies integrality of $a(\eta, \varpi)$. Putting everything together we get

Theorem 5.4. A pair $(\mathcal{C}, a)$ of an untwisted respectively twisted conjugacy class $\mathcal{C} \subset G$ and an $a \neq 0$ satisfies the integrality condition (2) if and only if $a \in \mathbb{Z} \backslash\{0\}$ and the natural symplectic structure $\frac{1}{2 \pi} \omega$ on the corresponding coadjoint orbit $\mathcal{O}_{(X, a)}$ of the loop group $L(G)$, respectively the twisted loop group $L(G, \tau)$ is integral.

The integrality condition for the Kirillov-Kostant form $\frac{1}{2 \pi} \omega$ on the coadjoint orbit $\mathcal{O}_{(X, a)}$ can be translated back to an explicit condition for the conjugacy class. Indeed, as we have seen in section 5.2, we can take $\frac{1}{a} X$ to be a constant $\frac{1}{a} X \in$ $\mathfrak{a} \subset \mathfrak{h} \subset \mathfrak{g} \subset L(\mathfrak{g})$ in the untwisted case and $\frac{1}{a} X \in \mathfrak{a}_{\tau} \subset \mathfrak{h}^{\tau} \subset \mathfrak{g}^{\tau} \subset L(\mathfrak{g}, \tau)$ in the twisted case. Now, the condition that $\frac{1}{2 \pi} \omega$ is an integral 2-form translates to the condition that $\alpha(X, a) \in \mathbb{Z}$ for all roots $\alpha$ of the (twisted) affine Lie algebra $L \widehat{\left(\mathfrak{g}_{\mathbb{C}}, \tau\right)}$. This gives again the condition that $a$ must be an integer. Furthermore, let us identify $\mathfrak{h} \oplus \mathbb{R}$ with its dual via the non-degenerate pairing from (3). Then, for fixed positive $a \in \mathbb{Z}$, the $(X, a)$ satisfying the integrality condition are exactly the highest weights of the irreducible highest weight representations of the (twisted) affine Lie algebra $\widehat{L(\mathfrak{g}, \tau)}$ at level $a$.

Remark 5.5. In fact, one can associate to each irreducible highest weight representation a coadjoint orbit of the corresponding (twisted) loop group [9, 19]. Under this correspondence, a highest weight representation with highest weight $(X, a)$ does not correspond to the coadjoint orbit passing through $(X, a)$ but rather to the orbit through $\left(X+\rho_{\tau}, a+h_{\tau}^{\vee}\right)$, where $\rho_{\tau} \in \mathfrak{h}^{\tau}$ denotes the projection of the half sum of all positive roots of $\mathfrak{g}_{\mathbb{C}}$ to $\mathfrak{h}^{\tau}$, and $h_{\tau}^{\vee}$ denotes the dual Coxeter number of the twisted affine Lie algebra $\widehat{L(\mathfrak{g}, \tau)}$ (in the untwisted case, just take $\tau=i d$. See e.g. [13] for more information on affine Lie algebras). 


\subsection{Proof of Proposition 5.3}

Before we start with the proof of Proposition 5.3, we need some preparations.

Lemma 5.6. For any $a \neq 0$, the map $\mathcal{O}_{(X, a)} \rightarrow \mathcal{C}$ which is given by $(Y, a) \mapsto$ $z_{(Y, a)}(2 \pi)$ is surjective.

Proof. First, note that the map $L(G, \tau) \rightarrow G$ which maps $\gamma \in L(G, \tau)$ to $\gamma(0)$ is surjective. Indeed, fix some $g \in G$ and choose $H \in \mathfrak{g}$ such that $\exp (H)=g$. We can decompose $\mathfrak{g}_{\mathbb{C}}=\bigoplus_{k=0}^{\operatorname{ord}(\tau)-1} \mathfrak{g}_{k}$, where $\mathfrak{g}_{k}$ denotes the $e^{2 \pi i \frac{k}{\operatorname{ord}(\tau)}}$-eigenspace of $\tau$. Viewing $H$ as an element of $\mathfrak{g}_{\mathbb{C}}$, we can write $H=\sum_{k=0}^{\operatorname{ord}(\tau)-1} H_{k}$. Let us set

$$
Y_{\mathbb{C}}(\theta)=\sum_{k=0}^{\operatorname{ord}(\tau)-1} H_{k} e^{\frac{i k}{\operatorname{ord}(\tau)} \theta}
$$

The real part $Y$ of $Y_{\mathbb{C}}$ is an element of $L(\mathfrak{g}, \tau)$ and we have $\exp (Y)(0)=g$.

Finally, fix some $g_{0} \in \mathcal{C}$. We can write $g_{0}=\tau(g) h g^{-1}$ for some $g \in G$ and $h \in G^{\tau}$. Fix $H \in \mathfrak{g}^{\tau}$ with $\exp \left(-\frac{2 \pi}{a} H\right)=h$, and $\gamma \in L(G, \tau)$ with $\gamma(0)=$ $g$. Set $Y_{0}(\theta)=\gamma(\theta) H \gamma(\theta)^{-1}-a \gamma^{\prime}(\theta) \gamma^{-1}(\theta)$. Then we have $z_{\left(Y_{0}, a\right)}(2 \pi)=$ $\gamma(2 \pi) \exp \left(-\frac{2 \pi}{a} H\right) \gamma(0)^{-1}=g_{0}$.

Proof of Proposition 5.3. Let us fix a triangulation $\left\{D_{i}\right\}_{i \in I}$ of $\partial N$ and a point $x_{i}$ in the interior of each triangle. If the triangulation is fine enough, each $D_{i}$ allows to choose a triangle $E_{i}$ in the Lie group $G$ such that the identity element $e \in G$ is in the interior of $E_{i}$ and we have $D_{i}=\left\{g x_{i} g^{-1} \mid g \in E_{i}\right\}$. Now, using the fact that the exponential map exp $: \mathfrak{g} \rightarrow G$ is locally invertible at $e$, we can refine the triangulation $\left\{D_{i}\right\}_{i \in I}$ to obtain triangles $E_{i}^{\prime}$ in $\mathfrak{g}$ with $0 \in E_{i}^{\prime}$ such that exp restricted to an open neighborhood of $E_{i}^{\prime}$ invertible, and $\exp \left(E_{i}^{\prime}\right)=E_{i}$. By the construction in the first part of the proof of Lemma 5.6, the triangles $E_{i}^{\prime}$ in $\mathfrak{g}$ give triangles $E_{i}^{\prime \prime} \subset L(G, \tau)$ in the loop group. Finally, using Lemma 5.6, for each $i \in I$ we can choose $X_{i} \in \mathcal{O}_{(X, a)}$ such that $z_{\left(X_{i}, a\right)}(2 \pi)=x_{i}$. Then the set

$$
D_{i}^{\prime}=\left\{\operatorname{Ad}_{\gamma}^{*}\left(X_{i}, a\right) \mid \gamma \in E_{i}^{\prime \prime}\right\} \subset \mathcal{O}_{(X, a)}
$$

is a triangle in $\mathcal{O}_{(X, a)}$.

Now the idea is to take the union of all $D_{i}^{\prime}$ with $i \in I$. But the triangles $D_{i}^{\prime}$ might not fit together to form a closed 2-cycle in $\mathcal{O}_{(X, a)}$. Indeed, if an element $g \in \partial N$ lies on the boundary of two triangles $D_{1}$ and $D_{2}$, the construction from above associates two elements $\left(Y_{1}, a\right)$ and $\left(Y_{2}, a\right)$ of $\mathcal{O}_{(X, a)}$ to $g$ which might be different. But we know that both $\left(Y_{j}, a\right)$ associated to such $g$ satisfy $z_{\left(Y_{j}, a\right)}(2 \pi)=g$. Set

$$
\mathcal{F}_{g}=\left\{(Y, a) \in \mathcal{O}_{(X, a)} \mid z_{(Y, a)}(2 \pi)=g\right\},
$$

and let us assume for the moment that $\pi_{0}\left(\mathcal{F}_{g}\right)=\pi_{1}\left(\mathcal{F}_{g}\right)=\{0\}$ for each $g \in G$.

Then, since $\mathcal{F}_{g}$ is connected, we can join $\left(Y_{1}, a\right)$ and $\left(Y_{2}, a\right)$ by a path inside $\mathcal{F}_{g}$. Furthermore, we can choose such a path for each point $g$ of the edge in such a way that it depends continuously on the point $g$. Indeed, we can consider the union of all $\mathcal{F}_{g}$ with $g$ an element of a fixed edge of the triangle $D_{i}$. This set is a subset 
of the coadjoint orbit and it is connected and simply connected (being a fibration over a closed interval with fibers isomorphic to $\mathcal{F}_{g}$ ). This shows that that one can join the edges continuously (by contracting the loop given by the edges together with the paths joining the endpoints of the edges).

Doing this for all edges of the triangulation $\left\{D_{i}\right\}_{i \in I}$, we can thus "join the edges" of the $D_{i}^{\prime}$. This procedure might still leave "holes" at the vertices of the triangulation. Let $g$ be a vertex of the triangulation. The boundary of a "hole at $g$ " is homeomorphic to an image of $S^{1}$ inside $\mathcal{F}_{g}$. But since we have assumed $\mathcal{F}_{g}$ to be simply connected, we can contract the boundary inside $\mathcal{F}_{g}$ and thereby "fill the hole". Repeating this process at each vertex of the triangulation, we obtain a closed 2-cycle in $\mathcal{O}_{(X, a)}$ with the desired properties.

So it remains to check that we indeed have $\pi_{0}\left(\mathcal{F}_{g}\right)=\pi_{1}\left(\mathcal{F}_{g}\right)=\{0\}$ for each $g \in G$. This is the content of the following lemma 5.7.

Lemma 5.7. We have $\pi_{0}\left(\mathcal{F}_{g}\right)=\pi_{1}\left(\mathcal{F}_{g}\right)=\{0\}$ for all $g \in G$.

Proof. Using the results of section 5.2 and denoting by $\operatorname{Stab}_{G}(g)$ the stabilizer of $g$ with respect to $\tau$-twisted conjugation, we can write

$$
\begin{aligned}
\mathcal{F}_{g} & =\left\{(Y, a) \in \mathcal{O}_{(X, a)} \mid z_{(Y, a)}(2 \pi)=g\right\} \\
& \cong\left\{\gamma \in L(G, \tau) \mid \gamma(0) \in \operatorname{Stab}_{G}(g)\right\} / \operatorname{Stab}_{L(G, \tau)}(X, a) .
\end{aligned}
$$

Again using the results described in section 5.2, we have $\operatorname{Stab}_{L(G, \tau)}(X, a) \cong$ $\operatorname{Stab}_{G}(g)$ via the map $\gamma \mapsto \gamma(0)$. Let us write $\mathfrak{G}_{g}=\{\gamma \in L(G, \tau) \mid \gamma(0)$ $\left.\in \operatorname{Stab}_{G}(g)\right\}$. Then we can use the long exact sequence

$$
\begin{aligned}
\cdots \rightarrow \pi_{1}\left(\operatorname{Stab}_{G}(g)\right) \rightarrow \pi_{1}\left(\mathfrak{G}_{g}\right) \rightarrow \pi_{1}\left(\mathcal{F}_{g}\right) \rightarrow & \\
& \rightarrow \pi_{0}\left(\operatorname{Stab}_{G}(g)\right) \rightarrow \pi_{0}\left(\mathfrak{G}_{g}\right) \rightarrow \pi_{0}\left(\mathcal{F}_{g}\right) \rightarrow 0
\end{aligned}
$$

to compute the homotopy groups. Indeed, it is easy to see that $\mathfrak{G}_{g}$ is connected so that $\pi_{0}\left(\mathcal{F}_{g}\right)=\{0\}$. Furthermore, since $G$ is simply connected and since $\operatorname{Stab}_{G}(g)$ is the fixed point set of an automorphism of $G$, we know that $\operatorname{Stab}_{G}(g)$ is connected. So if we can show that the injection $\iota: \operatorname{Stab}_{G}(g) \rightarrow \mathfrak{G}_{g}$ induces a surjection of fundamental groups, we are done.

Let $\varphi: S^{1} \rightarrow \mathfrak{G}_{g}, \theta \mapsto \varphi_{\theta}$ be a loop in $\mathfrak{G}_{g}$. The map $\theta \mapsto \varphi_{\theta}(0)$ defines a loop in $\operatorname{Stab}_{G}(g)$. Obviously, this map induces a surjection $\pi_{1}\left(\mathfrak{G}_{g}\right) \rightarrow \pi_{1}\left(\operatorname{Stab}_{G}(g)\right)$. It remains to show that the map is injective as well. So let $\varphi$ and $\widetilde{\varphi}$ be two loops in $\mathfrak{G}_{g}$ which map to the same element in $\pi_{1}\left(\operatorname{Stab}_{G}(g)\right)$. We have to show that $\varphi$ and $\widetilde{\varphi}$ are homotopic. Let $f$ be the loop in $\operatorname{Stab}_{G}(g)$ defined via $f(\theta)=\varphi_{\theta}(0)$ and accordingly $\widetilde{f}$. Assume that $\Phi:[0,1] \times[0,2 \pi]$ defines a homotopy from $f$ to $\widetilde{f}$. Then

$$
\widetilde{\Phi}:(s, \theta) \mapsto \varphi_{\theta} \iota(\Phi(0, \theta))^{-1} \iota(\Phi(s, \theta))
$$

defines a homotopy from $\varphi$ to a loop $\widehat{\varphi}$ in $\mathfrak{G}_{g}$ whose residual image $\widehat{f}: \theta \mapsto \widehat{\varphi}_{\theta}(0)$ equals $\widetilde{f}$. So from now on, we can assume that $f=\widetilde{f}$. Finally, we can use the fact that the set $\left\{\gamma \in L(G, \tau) \mid \gamma(0)=g_{0}\right\}$ is connected for each $g_{0} \in G$ to find a homotopy from $\varphi$ to $\widetilde{\varphi}$. Indeed, we can view the loops $\varphi$ and $\tilde{\varphi}$ as sections in a fibration over $S^{1}$, whose fiber at a point $\theta \in S^{1}$ is given by the set 
$\left\{\gamma \in L(G, \tau) \mid \gamma(0)=\varphi_{\theta}(0)\right\}$. One easily checks that the fibers are connected. Therefore, since $S^{1}$ is one-dimensional, the sections $\varphi$ and $\widetilde{\varphi}$ are homotopic. This finishes the proof.

Acknowledgements. We would like to thank A. Alekseev for helpful discussion and for pointing out the correct form of the integrality condition to us, and J. Fuchs and C. Schweigert for helpful remarks on an earlier version of this paper. The first author was supported by the Swiss National Science Foundation.

\section{References}

[1] Adams, J.F.: Lectures on Lie groups. W. A. Benjamin, Inc., New York, 1969

[2] Alekseev, A., Malkin, A., Meinrenken, E.: Lie group valued moment maps. J. Diff. Geom. 48, 445-495 (1998)

[3] Alekseev, A., Meinrenken, E., Woodward, C.: Duistermaat-Heckman measures and moduli spaces of flat bundles over surfaces. Geom. Funct. Anal. 12, 1-31 (2002)

[4] Alekseev, A., Schomerus, V.: D-branes in the WZW model. Phys. Rev. D 60, 06191 (1999)

[5] Birke, L., Fuchs, J., Schweigert, C.: Symmetry breaking boundary conditions and WZW orbifolds. Adv. Theor. Math. Phys. 3, 671-726 (1999)

[6] Bott, R.: An application of the Morse theory to the topology of Lie-groups. Bull. Soc. Math. France 84, 251-281 (1954)

[7] Bröcker, T., tomDieck, T.: Representations of Compact Lie Groups. Springer, Heidelberg, New York, 1985

[8] Felder, G., Fröhlich, J., Fuchs, J., Schweigert, C.: The geometry of WZW-branes. J. Geom. Phys. 34, 162-190 (2000)

[9] Frenkel, I.B.: Orbital theory for affine Lie algebras. Invent. Math. 77, 301-352 (1984)

[10] Fuchs, J., Schweigert, C.: Solitonic sectors, alpha-induction and symmetry breaking boundaries. Phys. Lett. B 490, 163-172 (2000)

[11] Gawedzki, K.: Conformal field theory: a case study, Conformal field theory (Istanbul, 1998). Front. Phys., 102, Adv. Book Program, Perseus Publ., Cambridge, MA, 2000

[12] Itoh, T., Sin, S.J.: Classification and auantum moduli space of D-branes in group manifolds. Phys. Lett. B 31, 126-134 (2002)

[13] Kac, V.G.: Infinite-dimensional Lie Algebras. 3rd edition, Cambridge University Press, Cambridge, 1990

[14] Meinrenken, E.: The basic gerbe over a compact simple Lie group. Preprint math.DG/0209194

[15] Mohrdieck, S.: Conjugacy classes of non-connected semi-simple algebraic groups. PhD thesis, Univ. Hamburg, 2000

[16] Pressley, A., Segal, G.: Loop Groups. Oxford University Press, Oxford, 1986

[17] deSiebenthal, J.: Sur le groups de Lie compactes non connexes. Comm. Math. Helv. 31, 41-89 (1956)

[18] Stanciu, S.: An Illustrated guide to D-branes in $S U_{3}$. Preprint hep-th/0111221

[19] Wendt, R.: Weyl's character formula for non-connected Lie groups and orbital theory for twisted affine Lie algebras. J. Funct. Anal. 180, 31-65 (2001) 